\title{
Heiner Busch
}

\section{Das polizeigrüne Europa \\ Warum eine „Rot-Grüne“ Regierung nicht viel verändert}

Herbst, Winter 1997: Am 26. Oktober 1997 sollten Italien, Österreich und Griechenland sich den sieben Staaten anschließen, die seit März 1995 das Schengener Durchführungsübereinkommen (SDÜ) anwenden. Der Vertrag sieht die Aufhebung der Kontrollen an den Binnengrenzen der beteiligten EU-Staaten vor. Dieser nach außen verkaufte politische Zweck des Vertrags sollte ausgeglichen werden - und zwar durch eine Verlagerung und Verschärfung der Kontrollen an den Außengrenzen, durch eine restriktive Asyl- und Visumspolitik, durch mehr polizeiliche Zusammenarbeit und das Kernstück - durch ein gemeinsam betriebenes elektronisches Fahndungssystem, das Schengener Informationssystem (SIS).

Der Termin des Beitritts konnte nicht gehalten werden. Seit September strandeten kurdische Flüchtlinge aus dem Nordirak an den Küsten Kalabriens. Die Innenminister der Schengen-Staaten, allen voran der deutsche Manfred Kanther, befürchteten, daß die ,illegalen Einwanderer“ nicht in Italien bleiben, sondern in den reicheren Norden der EU weiterwandern würden, wo bereits eine starke kurdische Gemeinde existiert. Italien, so hieß es, solle seine Grenzen sichern. Ab dem 1. Dezember durften die SchengenKandidaten zwar die Ausgleichsmaßnahmen mitmachen und wurden an das SIS angeschlossen. Der volle Beitritt und damit die Aufhebung der Grenzkontrollen erfolgte erst zum 1. April 1998. Italien war gezwungen, seine Flüchtlingspolitik zu verschärfen. Nach einer Polizeikonferenz in Rom, an der auch die Türkei teilnahm, bildete die Schengen-Gruppe eine Task-force, die polizeiliche Maßnahmen zur Abwehr der kurdischen Flüchtlinge erarbeiten soll. Auch auf Gesamt-EU-Ebene wurde unter maßgeblicher deutscher Beteiligung im Dezember 1997 ein Aktionsplan aufgelegt.

Manfred Kanther und seine CDU-Vorgänger im Bundesinnenministerium (BMI) haben in seltener Klarheit eine Politik vertreten, die etwas unscharf mit dem Schlagwort „Festung Europa“ bezeichnet wird: Abschottung nach außen, Härte nach innen. In ihre Amtszeit fallen die faktische Abschaffung des Grundrechts auf Asyl, die massive Ausdehnung der Befugnisse des Bundesgrenzschutzes, die personelle und technische Aufrüstung der deut- 
schen Ostgrenze, eines um das andere Sicherheitsgesetz, zuletzt der Grosse Lauschangriff. Law-and Order-Positionen vertraten sie nicht nur im Innern der BRD, sondern auch in den diversen europäischen Gremien.

Die Erwartung, daß sich diese Politik mit der Abwahl der CDU/FDPKoalition ändern würde, gehört in die Rubrik der schönen, nichtsdestoweniger falschen Hoffnungen, sprich: der Illusionen. Und das nicht nur, weil sich die ideologischen Positionen der Sozialdemokraten in Sachen Innere Sicherheit nicht wesentlich von denen der alten Regierungsparteien unterscheiden. SPD/FDP-Regierungen der 70er Jahre bauten das politische Strafrecht ebenso wieder aus wie Polizeien und Geheimdienste. Sämtliche großen polizeipolitischen Projekte und Sicherheitsgesetze der 80er und 90er Jahre wurden von der SPD im Bundestag und von den sozialdemokratischen Innenministern der Länder mitgetragen. Die GrundgesetzÄnderungen betreffend Asyl und Lauschangriff wären ohne die Stimmen der SPD gar nicht machbar gewesen. Über die Inneministerkonferenz und den Art. 23 GG waren die SPD-Länder durchgehend auch an der europäisierten Inneren Sicherheit beteiligt. Im Europa-Wahlkampf 1994 trat die Partei mit der Parole „Die Mafia in Europa bekämpfen“ auf. Das sozialdemokratische Europa-Konzept präsentierte sich mit einem Plakat, das mit Handschellen auf dem Rücken gefesselte Hände zeigte.

Spätestens der Blick in die Koalitionsvereinbarung der neuen Regierungsparteien hätte die Reformerwartungen in diesem Bereich herunterschrauben müssen. Denn in Sachen „Innere Sicherheit“ lautet dort die Parole: „wie sie sehen, sehen sie nichts." Weder für die nationale noch die europäisch gewendete Innere Sicherheit präsentierte die neue Regierung andere Konzepte. Tatsächlich hat sie auf EU- und Schengen-Ebene exakt da angeknüpft, wo die alte Regierung aufgehört hat. Die BRD übernahm am 1. Juli 1988 den Vorsitz im Schengener Exekutivausschuß. Ihre Präsidentschaft reichte bis zur Integration der Schengen-Kooperation in die EU, die mit dem Inkrafttreten des Amsterdamer Vertrages am 1. Mai 1999 vollendet wurde. Im ersten Halbjahr 1999 hatte die deutsche Regierung zusätzlich die Präsidentschaft in der EU - und damit auch im Rat der Innen- und Justizminister - inne. Eine solche Machtposition hat es in der EU-Innenpolitik bisher noch selten gegeben.

\section{Die Europafähigkeit von Sicherheitskalkülen gegen die Sperrigkeit demokratischer Forderungen}

$\mathrm{Da} ß$ diese Machtposition nicht für eine andere Politik genutzt wurde, liegt nicht nur an der ideologischen Nähe der sozialdemokratischen und der konservativen Staatsparteien in der EU, sondern auch am strukturellen Konservatismus der über den traditionellen Nationalstaat hinausreichenden Politik 
Innerer Sicherheit. Seit Mitte der 80er Jahre hat die innen- und justizpolitische Kooperation im Kontext der EU ein enormes Wachstum erlebt. Bei allen Schwierigkeiten der Verhandlungen im Detail zeigt sich, daß sich sicherheitspolitische Kalküle, die schließlich den Kernbestand der (national)staatlichen Souveränität betreffen, ausgezeichnet von der nationalen auf die supranationale Ebene transportieren lassen.

Das gilt sowohl für die alten und neuen Bedrohungsvorstellungen, als auch für die Konzepte, Instrumente und Methoden, mit denen auf diese Gefahren durch Polizei und zum Teil durch die Geheimdienste reagiert werden sollte. $\mathrm{Da}$ die Zusammenarbeit bei der Terrorismus-Bekämpfung am Anfang des neuen Schubs europäischer Polizeikooperation in den 70er Jahren stand, kann dabei nicht verwundern. Gemeinsame oder zumindest vermeintlich gemeinsame politische Gegner haben in der Geschichte der europäischen Polizeien schon immer die Zusammenarbeit - auch über politische Grenzen hinweg - erleichtert. Spätestens seit Mitte der 80er Jahre steht die „organisierte Kriminalität“" $(\mathrm{OK})$ und der Drogenhandel im Vordergrund der polizeilichen Debatten sowohl auf der nationalen Ebene der europäischen Staaten als auch in den diversen Gremien der internationalen und europäischen Kooperation. Beiden Phänomenen wird eine außerordentliche Gefährdung von Staat und Gesellschaft zugeschrieben, wobei die wahrgenommenen Gefahren nicht auf ein Staatsgebiet begrenzt sind. Forderungen nach einer verstärkten internationalen Zusammenarbeit begleiteten damit konsequenterweise sämtliche Diskussionen über den internationalen Terrorismus, die internationale organisierte Kriminalität oder den internationalen Drogenhandel. Sie scheinen geradezu unausweichlich - jedenfalls, wenn man akzeptiert, daß Polizei und Strafrecht die richtigen Mittel sind, um etwa das „Drogenproblem“ in den Griff zu bekommen.

Die Instrumente und Methoden der „Bekämpfung“ unterscheiden sich in den einzelnen Staaten nur wenig: es geht um eine Technisierung und Informatisierung, um eine Vorverlagerung des polizeilichen Eingriffs, insbesondere mit Hilfe verdeckter Methoden. Die internationale Zusammenarbeit der Polizei bewirkte dabei nicht nur eine Annäherung der Methoden und eine Kooperation im Einzelfall, sondern eine Internationalisierung der polizeilichen Tätigkeit selbst. Observationen und Einsätze von V-Leuten und verdeckten Ermittlern enden nicht mehr an Staatsgrenzen. Das Beispiel der kontrollierten Lieferung - einer Methode, die heute in der polizeilichen Drogenbekämpfung zum A\&O gehört - zeigt, wie die polizeilichen Handlungsweisen von vornherein grenzüberschreitend angelegt sind: Bei kontrollierten Lieferungen werden verbotene Waren - vor allem illegale Drogen - nach ihrer Entdeckung nicht sofort beschlagnahmt. Die Polizei läßt erkannte Transporte über die Grenzen hinweg bis zum Bestimmungsort laufen. Der Transport wird permanent observiert oder gar von innen heraus 
durch eingeschleuste V-Leute gesteuert. Mit der Beschlagnahme wird gewartet, bis sich Kaufinteressenten oder „Hintermänner“ präsentieren. Diese Methode markiert auch einen Wandel im polizeilichen Denken: Ziel ist nicht mehr, mit möglichst großen beschlagnahmten Mengen zu glänzen. Auf den kurzfristigen - nationalen - Erfolg wird verzichtet zugunsten des Versuchs, ganze Ringe oder Organisationen zu zerschlagen.

Die EU bzw. die Schengen-Gruppe boten einen politischen Rahmen für die nicht nur nationalstaatliche Verrechtlichung derartiger Formen der Zusammenarbeit - ein Prozeß, der nach wie vor im Gange ist. Sie ermöglichte weiter den Aufbau neuer gemeinsamer Institutionen wie Europol und gemeinsamer Datensysteme, weil sie im Unterschied zu anderen Zusammenhängen und Gremien einen tragfähigen politischen Rahmen hat bzw. ihn zumindest entwickeln konnte. Nationale und europäische Polizeientwicklung verliefen an diesen Punkten parallel - trotz der verschiedenen rechtlichen und organisatorischen Traditionen der beteiligten Nationalstaaten. Ähnliches gilt für die Asyl- und Ausländerpolitik und die damit verbundene Vereinheitlichung in Sachen Grenzpolizei. Bereits in den 70er Jahren begannen die Staaten des reichen europäischen Nordens, sich gegenüber Einwanderung und Flüchtlingen abzuschotten. Die Anwerbung von Arbeitskräften der 50er und 60er Jahre war vorbei. Trotz der durchaus vergleichbaren Politik, die hier betrieben wurde, zog man nicht am gleichen Strang. Ganz im Gegenteil bedeutete die nationalstaatliche Abschottungspolitik bis Mitte der 80er Jahre, daß die einzelnen Staaten versuchten, Flüchtlinge in Nachbarstaaten abzudrängen. Die danach begonnene „Harmonisierung des Asylrechts" führte nicht zu einer gemeinsamen Aufnahmepolitik. Diese gibt es, wie das Schicksal der Kosovo-Flüchtlinge gerade wieder demonstrierte, immer noch nicht. Vielmehr versuchte man sich in einer gemeinsamen Abschottung, einer Festlegung gemeinsamer Standards einer rigiden Kontrolle der EU- bzw. Schengen-Außengrenzen, einer vereinheitlichten Visumspolitik, eines Systems von Rückübernahmeabkommen, mit dem auch die umliegenden Nicht-EU-Staaten zur Anpassung gezwungen wurden. Der erste Schritt dahin war die Einigung auf das Prinzip, daß pro Flüchtling nur ein Asylantrag in der EU gestellt werden darf - und zwar in der Regel in dem Staat, wo die Person erstmals EU-Boden betritt. Nachfolge- oder Parallelanträge wurden durch das SDÜ bzw. das inhaltlich gleiche Dubliner Abkommen ausgeschlossen. Der jetzt definitiv anstehende Aufbau einer gemeinsamen Datenbank für Fingerabdrücke von Flüchtlingen und ,illegalen Einwanderern“ Eurodac ist eine der Konsequenzen. Während polizeiliche Bedrohungsvorstellungen und Bekämpfungskonzepte sehr wohl auf eine supranationale Ebene transferiert werden konnten, sind Fragen der Kontrolle polizeilicher Macht, des Datenschutzes oder des Ausbaus der Rechte von Flüchtlingen und MigrantInnen sperrig. 
Der Datenschutz im Polizeibereich erreicht auf EU-Ebene allenfalls einen rhetorischen Stellenwert. Rechte auf Auskunft über die zur eigenen Person gespeicherten Daten sind z.B. in der Europol-Konvention enthalten, aber mit so vielen Hindernissen ausgestaltet, daß die Verweigerung der Auskunft immer wahrscheinlicher ist, als deren Gewährung. Nicht einmal diese Rhetorik ist unter den EU-Staaten unumstritten. Entsprechende Formulierungen wurden vor allem von deutscher Seite in den diversen Abkommen durchgesetzt. Bei genauerer Betrachtung der verschiedenen EU-Abkommen zeigt sich, daß diese den neueren deutschen Polizeigesetzen sehr ähneln. Auch wenn die Datenweitergabe und -auswertung unbegrenzt ist, so wird diese doch bis ins einzelne verrechtlicht. Das Volkszählungsurteil des Bundesverfassungsgerichts vom Dezember 1983 zwingt die deutschen Gesetzgeber jeweils eine gesetzliche Grundlage zu schaffen, wenn Zweckbindungen von Daten aufgehoben werden. Das Ergebnis dessen ist die Aufblähung von Gesetzes- bzw. Abkommenstexten sowie die Praxis der „Scheinvertatbestandlichung“. Das Wortungetüm bedeutet, daß polizeiliche Aufgaben und Befugnisse als Tatbestand gefaßt werden, daß aber eine Grenze zum Illegalen nicht mehr erkennbar ist. Die Gesetze oder Abkommen ermächtigen die Polizei, aber sie verbieten ihr kaum mehr etwas.

Die liberale Rechtskultur, das Set von Institutionen und Regeln zur Einhegung staatlicher Macht, ein gewisses Mindestmaß an parlamentarischer Kontrolle und vor allem die Wachsamkeit einer Öffentlichkeit ist angesichts wirksam vorgetragener Feindbilder und Bedrohungsvorstellungen schon im Nationalstaat ausgehöhlt worden. Auf supranationaler Ebene ist ein solcher Set aber gar nicht vorhanden. Der Blick auf die organisatorische Entwicklung der innen- und justizpolitischen Zusammenarbeit im Kontext der EU zeigt, daß diese fast ausschließlich exekutivisch dominiert ist.

\section{Ein Blick auf die organisatorische Entwicklung}

\section{Die Anfänge}

Bis Mitte der 70er Jahre hatte das EWG-Europa der damals zehn Mitgliedsstaaten nichts mit polizeilichen Fragen am Hut. Die E(W)G war eine Wirtschaftsgemeinschaft - jedenfalls bis zur Gründung von TREVI im Jahre 1976. Das Kürzel TREVI stand für ,terorisme, radicalisme, extremisme, violence internationale" und verweist damit gleichzeitig auf Zweck und Anlaß der Kooperation, die Bekämpfung politisch motivierter Kriminalität einschließlich des vermuteten Um- und Vorfeldes. Das Gremium wurde bewußt außerhalb der Strukturen der EG gehalten und sollte eine informelle Zusammenarbeit ermöglichen. Seine Organisation, wenn man von einer solchen in den ersten Jahren überhaupt reden kann, war die typische Struktur einer Regierungskooperation. Auf der oberen Ebene die Minister, dar- 
unter die Runde der Hohen Beamten - sprich der Staatssekretäre - und unter ihnen die Arbeitsebene. Diese bestand im ersten Jahrzehnt in zwei Arbeitsgruppen: TREVI I - Terrorismus, TREVI II - Technik und Ausbildung. Nur in der ersten Arbeitsgruppe fand tatsächlich eine praktische Ermittlungskooperation zwischen den nationalen Verbindungsbüros statt, die entweder bei den Inlandsgeheimdiensten oder den Zentralen der politischen Polizeien angesiedelt waren.

Zwar hatte die Zusammenarbeit im Rahmen von TREVI eine bedeutende Koordinationsfunktion und eine nicht zu unterschätzende politische Wirkung. Bindende Beschlüsse konnte das Gremium aber keine treffen. Erst ab Mitte der 80er Jahre ist ein Ausbau der Kooperation festzustellen. Eine weitere Arbeitsgruppe entstand: TREVI III - Organisierte Kriminalität/ Drogen. Die organisatorische Struktur wurde gefestigt. Treffen fanden nun häufiger statt. Zusätzlich zur Präsidentschaft, die parallel zum Vorsitz in der EG alterniert, wurde eine Troika aus der gegenwärtigen, der vergangenen und der kommenden Präsidentschaft gebildet, die für mehr Kontinuität sorgen sollte. Parallel zu ihren TREVI-Sitzungen trafen sich die Minister ab 1986 auch als „EG-Einwanderungsminister“. Neben TREVI entstand die Ad hoc-Gruppe „Einwanderung“ mit entsprechenden weiteren Untergruppen auf der Arbeitsebene. Die vorhandene lose Struktur der innen- und justizpolitischen Kooperation erhielt außerdem den Auftrag, die Folgerungen aus dem für den 31.12.1992 projektierten EG-Binnenmarkt - d.h. der Aufhebung der Personenkontrollen an den Binnengrenzen der Gemeinschaft auf polizeilichem Gebiet zu erarbeiten. Auf diesem Gebiet hatten die Regierungen der Mitgliedstaaten allesamt eine Zuständigkeit der EGKommission und damit einen minimalen Einfluß des Europäischen Parlaments (EP) verhindert.

Der Blueprint für das polizeiliche Binnenmarkt-Europa kam aber nicht von TREVI, sondern aus dem polizeilichen Kerneuropa: In Schengen einigten sich die Innen- bzw. Justizminister der Benelux-Staaten, der BRD und Frankreichs 1985 auf ein erstes Abkommen: Es stellte die mit dem Binnenmarkt verbundene Aufhebung der Binnengrenzkontrollen in Aussicht, verlangte aber gleichzeitig den Ausgleich des angeblich zu erwartenden Sicherheitsverlustes. Beides sollte in einem zweiten Abkommen - dem Durchführungsübereinkommen - fixiert werden. Damit entstand ab 1985 eine Arbeits- und Verhandlungsstruktur außerhalb der schon bestehenden TREVI-Kooperation. Mit dem ohnehin falschen Deal - Grenzöffnung, dafür Ausgleichsmaßnahmen - fixierte die Gruppe nicht nur ein ausgesprochen wirksames ideologisches Muster, sondern setzte im 1990 unterschriebenen SDÜ auch die polizei- und ausländerpolitischen Standards. Mit Ausnahme Großbritanniens und Irlands schlossen sich nach und nach alle Mitgliedsstaaten dem SDÜ an. 
Auf der Ebene der damals 12 EG-Staaten waren die Verhandlungen dagegen blockiert. Insbesondere mit Großbritannien und der Republik Irland war und ist eine Aufhebung von Binnengrenzkontrollen nicht zu machen. Ende 1988 setzten die Staats- und Regierungschefs der EU, der Europäische Rat, die Gruppe der Koordinatoren „Freizügigkeit“ ein. Sie sollten die Verhandlungen koordinieren und doch noch dafür sorgen, daß der 31.12.1992 als Termin für die Aufhebung der Binnengrenzkontrollen eingehalten werden könnte. Bereits nach kurzer Zeit präsentierten sie das Palma-Dokument. Solche Listen von zu erreichenden Zielen, die gleichzeitig Termine für die Realisierung und das jeweils dafür verantwortliche Gremium benannte, wurden in der Folge zur gängigen Praxis. Das einzige Abkommen, das bis zum Binnenmarkt-Termin ausgehandelt wurde, ist das Dubliner Abkommen, das dieselbe Erstasyl-Regelung beinhaltet wie das SDÜ. Bis zum Inkrafttreten des Maastrichter Vertrages, der die gemeinsame Innenpolitik auf eine neue Ebene hob, hatte man sich allerdings auf eine ganze Reihe von Punkten grundsätzlich geeinigt: auf Europol, auf die Schaffung eines Zollinformationssystems, auf die Ausdehnung des SIS zum Europäischen Informationssystem EIS, etc.

\section{Maastricht}

Der Maastrichter EU-Vertrag - 1992 unterzeichnet, Ende 1993 in Kraft getreten - machte die Innen- und Justizpolitik zu einer Angelegenheit gemeinsamen Interesses. An die Stelle der informellen Regierungskooperation in TREVI trat nun eine formelle. Neben der ersten Säule, der eigentlichen Gemeinschaft und dem Binnenmarkt, und der zweiten - der gemeinsamen Außen- und Sicherheitspolitik - wurde die Innen- und Justizpolitik zur dritten Säule der Union erhoben.

Organisatorisch bewirkte der Vertrag eine Integration der verschiedenen auf EG- bzw. nun EU-Ebene existierenden Arbeitsgruppen und Foren: Die Koordinatoren Freizügigkeit wurden zum K4-Ausschuß (benannt nach Art. K4 EUV). Die Ad-hoc-Gruppe Einwanderung wurde mit ihren diversen Untergruppen zur Lenkungsgruppe I. TREVI und die Groupe d'Assistance Mutuelle (GAM) der Zollverwaltungen wurden zur Lenkungsgruppe II zusammengefaßt. Auch hier blieben die diversen Unterguppen erhalten. Ebenfalls in diese Struktur integriert wurde eine Lenkungsgruppe III - Kooperation auf dem Gebiet des Zivil- und des Strafrechts.

Die dritte Säule produzierte aber im Unterschied zur ersten keine Richtlinien und Verordnungen, bei deren Zustandekommen je nach Rechtsgrundlage das EP mehr oder weniger beteiligt wird, sondern Rechtsinstrumente, die typisch sind für die Zusammenarbeit souveräner Staaten: Standpunkte und Resolutionen, die allerdings unverbindlich bleiben, Gemeinsame Maß- 
nahmen, die auf bestehendes nationales oder Vertragsrecht abgestützt werden, und schließlich völkerrechtliche Abkommen. Bei alledem wurde das EP allenfalls informiert, wobei der Grad der Information vom guten Willen der Minister und des K 4-Ausschusses abhängig war.

Standpunkte und Maßnahmen kamen relativ einfach zustande. Trotz ihres unverbindlichen Charakters bewirkten sie eine nicht zu unterschätzende Angleichung der jeweiligen nationalen Politik. Verhandlungen von Abkommen waren dagegen langwierig. Vom Verhandlungsbeginn bis zum Abschluß des Prozesses der Ratifikation durch die Parlamente der Mitgliedsstaaten ist es ein langer Weg, der im Falle der Europol-Konvention insgesamt sechs Jahre betrug.

Neben der dritten Säule der EU blieb die Schengen-Kooperation erhalten und bildete weiter für viele Fragen den Motor für die gesamte EU: Der Schengener Exekutivausschuß, der die aus den Vertragsverhandlungen übernommenen Arbeitsgruppen in eine ständige Arbeitsstruktur überführte, produzierte Standards der Außengrenzkontrolle, „Handbücher“ für diverse Formen der Zusammenarbeit; er gab Impulse für eine Vielzahl bilateraler Polizeiabkommen; er wurde zum organisatorischen Rahmen für eine praktische Kooperation der Polizeien, die weit über den Inhalt des SDÜ hinausgeht (z.B. Pilotprojekte für verdeckte Ermittlungen u.ä.).

\section{Amsterdam}

Ab 1996 befaßte sich eine Regierungskonferenz mit der Erneuerung des Maastrichter Vertrages, deren Ergebnis der Amsterdamer Vertrag ist. Die großen Hoffnungen, die selbst kritische Euro-ParlamentarierInnen auf diesen Vertrag setzten, wurden aber enttäuscht. Im Bereich der Innen- und Justizpolitik bewirkt der Vertrag eine partielle Vergemeinschaftung: Fragen der Asyl- und Einwanderungspolitik sowie der Aussengrenzen wanderten aus der dritten in die erste Säule. Während der ersten fünf Jahre nach Inkrafttreten soll der Rat allerdings weiterhin alle Entscheidungen einstimmig fassen. Die Kommission erhält Initiativrecht, d.h sie dürfte sich im weiteren als zusätzliche Kraft auf diesem Gebiet etablieren. Statt Abkommen werden in diesem Segment der Kooperation jetzt Verordnungen und Richtlinien produziert. Diese brauchen nicht mehr von den nationalen Parlamenten ratifiziert werden, allerdings wird auch das Europaparlament (EP) nur angehört. D.h. es darf sich äußern, was es sagt, hat aber für die Entscheidung des Rates keinen weiteren Einfluß. Was das heißt, zeigt sich derzeit an den Verhandlungen über Eurodac. Hier lagen bereits Mitte letzten Jahres ein fertiger Entwurf für ein Abkommen und ein zusätzliches Protokoll vor. Statt das Paket zu unterzeichnen, wurde es bis zum Inkrafttreten des Amsterdamer Vertrags am 1. Mai dieses Jahres eingefroren. Bereits Ende Mai 
wartete die Kommission mit einem Verordnungsentwurf auf, der im wesentlichen den Inhalt des Abkommens und des Protokolls aufgreift. Das EP kann sich jetzt äussern. Da der Rat sich letzten Endes schon festgelegt hat, ist aber bereits jetzt absehbar, daß das Votum des Parlaments kaum Einfluß haben wird. Da die Verordnung nicht mehr von einer Ratifikation der nationalen Parlamente abhängt, könnte Eurodac schon in Kürze Wirklichkeit werden.

Zoll, Polizei und Strafrecht bleiben auch nach dem Amsterdamer Vertrag Gegenstände bloßer Regierungszusammenarbeit. Auch hier darf das Parlament Stellung nehmen. Das war's dann aber. Aus den Erfahrungen mit der Maastrichter dritten Säule hat man insofern gelernt, als Mechanismen der Beschleunigung eingeführt wurden. So sollen Abkommen bereits in Kraft treten, wenn sie von der Hälfte der Mitgliedsstaaten ratifiziert wurden. Neben Abkommen und Gemeinsamen Maßnahmen können nun auch Rahmenbeschlüsse verabschiedet werden. Während die Maßnahmen auf bestehendem Recht beruhen mußten, schaffen Rahmenbeschlüsse neues Recht. Sie sind - ähnlich wie Richtlinien auf der Gemeinschaftsebene - für die Mitgliedsstaaten verbindlich und müssen ins nationale Recht überführt werden. Auf welche Weise dies geschieht, steht den Mitgliedsstaaten frei. Die Schengen-Kooperation wird nun in die EU überführt: Sowohl die Abkommen als auch die ca. 170 Beschlüsse des Exekutivausschusses mußten auf ihre Zugehörigkeit zur neuen ersten bzw. dritten Säule überprüft werden. Das SIS wurde als ganzes der dritten Säule und damit der Verantwortung der Exekutiven zugeordnet. Großbritannien und Irland können Teile des Schengen-Acquis übernehmen. Sie werden voraussichtlich dem SIS beitreten, aber die Aufhebung der Binnengrenzkontrollen verweigern.

\section{Exekutivlastigkeit}

Wer trägt also die Entscheidungen der EU-europäischen Politik Innerer Sicherheit? Antwort: Minister, hohe Ministerialbeamte und die Repräsentanten von Polizei, Zoll und Strafverfolgungsbehörden der Mitgliedsstaaten. Eine parlamentarische Kontrolle gab es bisher so gut wie nicht. Das EP war systematisch ausgeschlossen. Es konnte sich äußern und hat das auch zum Teil durchaus liberal getan, wobei die - unverbindlichen - Beschlüsse und Berichte des EP in dem Maße realpolitischer wurden, da die Chance auf eine Erweiterung seines Mandats bestand.

Aber auch die nationalen Parlamente sind faktisch machtlos. Nur ein Teil der Entscheidungen des Rates oder des nunmehr in die Ratsstruktur eingegliederten Schengener Exekutivausschusses werden überhaupt von den nationalen Parlamenten zur Kenntnis genommen bzw. ihnen unterbreitet. Dabei war der Bundestag schon in der letzten Legislaturperiode vergleichs- 
weise gut bedient. Zumindest die fertigen Beschlüsse legte man dem Innenausschuß bzw. seinem Berichterstattergremium vor.

Die Plenarversammlungen der Parlamente befassen sich mit diesen Fragen allenfalls dann, wenn ein Abkommen eben ratifiziert werden muß. Auch Abkommen werden aber erst dann zum Thema der Parlamente, wenn der Ratifizierungstermin wirklich ansteht. Dann allerdings sind die wesentlichen Entscheidungen bereits gefallen. Die parlamentarische Demokratie erschöpft sich hier im Hände-Hoch-Verfahren. Wie das funktioniert, zeigte die Debatte um das SDÜ: Schon 1988 standen die grundlegenden Entscheidungen in Form von Vorentwürfen fest. Der Rest waren Details. 1990 wurde unterzeichnet. In Deutschland folgte die Verabschiedung nach der Abschaffung des Asylrechts 1993. In der Zwischenzeit hatte der Exekutivausschuß schon einen großen Teil der in den „Handbüchern“ fixierten Ausführungsbestimmungen beschlossen. Der Aufbau des SIS war in vollem Gange. Über die Ausführung des Abkommens, die schon vor der Ratifizierung erfolgte, wurde natürlich nicht abgestimmt. Weil alles schon vorbereitet war, hätte der Bundestag sich einen Rückzug nie leisten können.

Noch deutlicher das Beispiel Europol: Schon in den 70er Jahren gab es auf Seiten der Polizei einige Forderungen nach einer zentralen europäischen Polizeibehörde, die allerdings chancenlos waren, weil der EWG damals das notwendige politische Gerüst fehlte. Auch 1988, als Kanzler Kohl diese Forderungen wieder aufgriff und auf einer Tagung der Staats- und Regierungschefs ein Europäisches FBI forderte, stieß er bei seinen Kollegen auf eine abwehrende Haltung. Zentrale supranationale Informationssammlung und Ermittlungsbefugnisse für ein zentrales Amt wären zum Beispiel für die Niederlande damals undenkbar gewesen. 1991 trug Kohl die Forderung noch einmal vor, diesmal mit Erfolg. Inzwischen wurde im Rahmen der dritten TREVI-AG der britische Vorschlag einer European Drugs Intelligence Unit gehandelt. Der Europäische Rat beschloß also die Errichtung Europols, allerdings nur als Informationsdrehscheibe für die nationalen Polizeien. Die TREVI-Minister setzten den Beschluß um. 1992 fand er Eingang in den Maastrichter Vertrag. Mitte 1993 beschlossen die TREVIMinister eine „ministerielle Vereinbarung“. Wegen der absehbaren Länge der Verhandlungen und der folgenden Ratifikation sollte zunächst ein Europol-Vorläufer aufgebaut werden, die Europol Drugs Unit (EDU). Diese sollte sich nur mit der Bekämpfung des Drogenhandels befassen und auch nur auf der Basis nationalen Rechts und bestehender internationaler Abkommen arbeiten dürfen. Eine Farce: Die EDU bekam zwar keine eigenen Datenverarbeitungsanlagen, aber die nach Den Haag entsandten Verbindungsbeamte der nationalen Polizeien erhielten Zugang zu ihren jeweiligen nationalen Informationssystemen, aus denen sie nach eigenem Ermessen Daten an die Verbindungsbeamten der jeweils anderen Staaten weitergeben 
konnten. Sie repräsentieren quasi die Gesamtheit der Befugnisse ihres Heimatstaates. Die EDU besorgte nicht nur eine schnelle Vermittlung von Informationen, sondern betrieb bereits gemeinsame Analysen. Die Verbindungsbeamten steuerten und koordinierten Kontrollierte Lieferungen und andere Operationen.

Als im Januar 1994 dieses „Provisorium“ die Arbeit aufnahm, war damit ohne jegliche Parlamentsentscheidung und ohne Abkommen der Aufbau eines großen Teils von Europol realisiert. Die Zahl der von diesem Provisorium abgewickelten Anfragen stieg stetig. 1995 und 1996 wurde - ebenfalls durch bloßen Ministerentscheid, durch eine Gemeinsame Maßnahme das Mandat der Drogeneinheit erweitert: um Kfz-Diebstähle, „Schleuserkriminalität", Nuklearschmuggel und schliesslich um Menschenhandel. Erst Mitte 1995 wurde das Abkommen unterzeichnet. Über die minimale Beteiligung des Europäischen Gerichtshofes wurde ein Formelkompromiß in Form eines Protokolls erstellt, das Großbritannien faktisch von allen Verpflichtungen ausnimmt. Die Unterzeichnung bedeutete auch den Startschuß für die Arbeiten zur Vorbereitung der gemeinsamen Informationssysteme. Als der Ratifizierungsprozeß am 1. Oktober 1998 abgeschlossen war, fehlten nur noch Details der Ausführungsbestimmungen sowie ein Knopfdruck, der einen zentralen Bestandteil der gemeinsamen Informatik in Betrieb setzte. Bereits im Amsterdamer Vertrag, der im Frühjahr 1997 unterzeichnet worden war, hatte man sich aber auf die Ausdehnung der Kompetenzen von Europol geeinigt: Das Amt sollte nicht nur Informationen sammeln und verarbeiten, sondern auch operative Befugnisse erhalten. Die Europol-Konvention war damit schon wieder für inaktuell erklärt worden, noch bevor sie in Kraft war. Wie diese neuen Befugnisse rechtlich verankert werden, ist noch unklar. Deutlich wird aber, daß die Parlamente ständig hinterherhinken. Sie

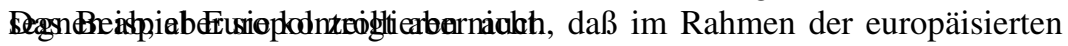
Inneren Sicherheit ein ständiges Mehr entsteht. Gerade die Schwierigkeit, im Detail Ergebnisse auszuhandeln, macht es faktisch undenkbar, hinter das Ausgehandelte zurückzufallen. Kein Minister und keiner seiner Bürokraten würde akzeptieren, daß ausgehandelte Kompromisse neu aufgerollt würden. Dafür daß dies nicht passiert, hat sich auf EU-Ebene ein eigener Mechanismus herausgebildet: der Acquis. Das „Erworbene“ wird fixiert. Dies war so im Falle von TREVI. Dies war so bei Schengen, wo der Acquis sowohl die Abkommen als auch die Beschlüsse des Exekutivausschusses beinhaltete. Dies betrifft die EU-Zusammenarbeit als Ganze, aber auch einzelne Bereiche, für die Unter-Acquis bestehen. Und diese Acquis werden auch den Beitrittskandidaten entgegengehalten. Die jeweiligen Beschlüsse sind nicht mehr wegzudenken. Es gibt nur noch einen Weg nach „,vorne“, d.h. zu mehr und neuen Instrumenten der Zusammenarbeit, aber 
keinen zurück. Die Beitrittsstaaten haben sogar die schon abgeklärten Teile von Abkommensentwürfen zu akzeptieren.

Aktionspläne sorgen ferner dafür, daß die Schraube sich weiter dreht - mit Beschleunigungseffekt. So wurde Ende 1996, Anfang 1997 ein EUAktionsplan zur Bekämpfung der organisierten Kriminalität ausgehandelt. In ganzen vier Monaten erstellte eine „Hochrangige Gruppe OK“ diesen Forderungskatalog. Dafür, daß er umgesetzt wird, sorgt die Gruppe mittlerweile selbst. Sie ließ sich vom Europäischen Rat in „Multidisziplinäre Gruppe OK" umtaufen und legt nun alle halbe Jahre Sachstandsberichte vor. Der Ministerrat beschloß in einer gemeinsamen Maßnahme vom Dezember 1997 ferner Evaluierungsmechanismen, mit denen die Mitgliedsstaaten daraufhin überprüft werden, ob sie ihre Verpflichtungen in Sachen OK-Bekämpfung auch einhalten.

Gegenüber der nationalen Politik erscheint Europa immer mehr als ein dominierender Faktor. Das zeigt sich nicht nur daran, daß auf supranationaler Ebene von den Exekutiven ausgehandelte Abkommen oft über das hinausgehen, was auf nationaler Ebene Recht ist. ${ }^{1}$ Die europäisch versammelte Exekutive beschließt sogar zu überprüfen, ob sie und ob die Parlamente auf nationaler Ebene ihre Pflicht getan haben.

\section{Pflicht zum langen Atem}

Die EU hat sich zu einem Staatsgebilde besonderer Art entwickelt. Als staatliche Einheit wird sie nicht nur von denen wahrgenommen, die als Flüchtlinge oder Einwanderer ihre Grenzen überschreiten wollen. Als Staat kristallisiert sie sich auch in ihren polizeilichen Machtmitteln heraus. Entschieden wird allerdings in einer Form, die in weiten Teilen noch die der zwischenstaatlichen Kooperation souveräner Regierungen ist. Verglichen mit dem liberalen Nationalstaat, an den wir uns gewöhnt haben, fehlen hier weitgehend die Mittel, an denen eine Opposition ansetzen könnte. Während man bei aller Schwäche im Nationalstaat die Regierung immerhin identifizieren und entsprechend politisch angreifen kann, ist die politische Macht in diesem neuen Staatsgebilde weitgehend ortlos. Eine Öffentlichkeit zu organisieren, ist nicht nur deshalb schwer, weil Brüssel so weit entfernt ist, sondern weil der Gang von Entscheidungen auf europäischer Ebene undurchsichtig ist. Opposition scheitert daran, daß ihre potentiellen nationalen

1 Nach wie vor deutlichstes Beispiel dafür war die Diskussion um das Asylrecht 1993. Hier argumentierte der damalige Innenminister Schäuble, daß das Grundrecht auf Asyl fallen müsse, damit Deutschland an den in Schengen und in der EU getroffenen Entscheidungen teilnehmen könne. Auf europäischer Ebene hatte die Bundesregierung aber nicht nur die Entscheidungen mitgetragen, sondern sie selbst aktiv herbeigeführt. Sie verhandelte konträr zum nationalen Verfassungsrecht. Sie entschied damit die Ergebnisse der Debatten des Bundestages vor. 
Bestandteile die unterschiedlichsten Positionen vertreten. Selbst schwedischen Linken sind Forderungen nach einer Entkriminalisierung oder gar Legalisierung von Drogen fremd. Schon schwedische Liberale sind dagegen für Forderungen nach mehr Transparenz zu gewinnen, die etwa französischen Kommunisten nicht geläufig sind. Die Fraktionen im EP lassen sich deshalb kaum mit denen auf nationaler Ebene vergleichen. Die praktischen Schwierigkeiten beginnen aber oft bereits da, wo es um Übersetzungsmöglichkeiten, um das Arrangement von Tagungen o.ä. geht. Gegenüber der Macht der Exekutive sind die oppositionellen Gehversuche oft geradezu hilflos.

War die Politik Innerer Sicherheit auf nationaler Ebene schon ein Fall bürokratisierter Politik, so ist sie das auf EU-Ebene um so mehr. Daß sich hier durch einen Regierungswechsel schnell etwas ändert, ist kaum zu erwarten. Noch dazu, wenn der kleine Partner in einer nationalen Regierung, der etwas zu ändern bereit wäre, so schwach ist wie die Grünen in der neuen Regierung. Über diese mageren Chancen mag man enttäuscht sein, eine Argumentation nach dem Motto: ,wer hat uns verraten? ...." bringt aber nicht viel. Die auf längere Sicht bestehende Machtlosigkeit bürgerrechtlicher und demokratischer Argumente ist ein Faktum, das man zunächst einmal zur Kenntnis nehmen muß. Ärgerlich wird es allerdings dann, wenn diese Machtlosigkeit dadurch rationalisiert wird, daß richtige Forderungen wie Ballast über Bord geworfen werden.

Die Linke und die Bürgerrechtsgruppierungen - dazu gehört zumindest ein Teil der grünen Regierungspartei - müssen einen langen Atem haben. Sie müssen nicht nur das - gelinde gesagt - „Demokratiedefizit“ der EU zum Gegenstand ihrer Kritik machen. Sie haben darüber hinaus öffentlich klarzumachen, daß der Aufbau von immer neuen Polizeiinstitutionen und Datenbanken, daß mehr polizeiliche Kooperation nichts anderes sind als ein Verzicht auf politische Gestaltung. Für diese Arbeit, die notwendigerweise zu einem großen Teil außerparlamentarisch geleistet werden muß, braucht es Radikalität. Realpolitik bewegt hier nichts.

\section{Literatur}

Aden, Hartmut (1998): Polizeipolitik in Europa, Opladen (Westdeutscher Verlag).

Bigo, Didier (1996): Polices en réseaux. L'expérience européenne, Paris (Presse de la FSNP). Innere Sicherheit in der EU (1996): Bürgerrechte \& Polizei/ CILIP 53, 1/ 1996.

Europas neue Grenzen (1998): Bürgerrechte \& Polizei/ CILIP 59, 1/1998.

Statewatch-Bulletin, erscheint sechsmal jährlich in London (PO Box 1516, London N 16 0EW

Busch, Heiner (1995): Grenzenlose Polizei. Neue Grenzen und polizeiliche Zusammenarbeit in Europa, Münster (Westfälisches Dampfboot).

Busch, Heiner (1999): Polizeiliche Drogenbekämpfung - eine internationale Verstrickung, Münster (Westfälisches Dampfboot). 